Artículo Corto

\title{
Composición nutricional del tallo de Silbadero (Geoffroea spinosa Jacq.) del municipio de Tubara, Atlántico
}

\author{
Nutritional composition of Silbadero stem (Geoffroea spinosa Jacq.) of the \\ municipality of Tubara Atlantico
}

\author{
Amparo L. Púa ${ }^{1 *}$, Genisberto E. Barreto ${ }^{2}$, Milagros D. De La Hoz ${ }^{3}$, Yulieth P. Fritz ${ }^{3}$ \\ Recibido para publicación: Octubre 24 de 2016 - Aceptado para publicación: Mayo 18 de 2017
}

\begin{abstract}
RESUMEN
El Silbadero (Geoffroea spinosa Jacq) es una leguminosa que se encuentra en países suramericanos como Chile, Argentina, Bolivia, Uruguay y Colombia, de esta especie solo se han reportado estudios taxonómicos y fitoquímicos. El objetivo del presente estudio fue determinar el contenido de nutrientes del tallo de la especie vegetal Silbadero. Las muestras de la especie fueron tomadas al azar mediante una salida de campo a la finca los Tres ubicada en Tubará, Atlántico. Se determinaron los parámetros de proteínas, grasas, carbohidratos, fibra bruta, cenizas, humedad; el aminoácido Lisina; minerales como el zinc, calcio, hierro y magnesio y vitaminas del complejo B (Tiamina, Riboflavina, Niacina, ácido Fólico). Las proteínas fueron determinadas por el método Kjeldahl, las grasas por el método Soxhlet, los carbohidratos por diferencia, fibra bruta por el método ácido-base, cenizas por incineración, humedad por gravimetría; la Lisina por cromatografía ultra rápida; los minerales por espectrofometría de absorción atómica y la vitaminas por cromatografía líquida de alta resolución. El contenido de proteína fue de 4,56\%, grasas $0,38 \%$, carbohidratos $37,2 \%$, fibra bruta $43,5 \%$, cenizas $7,6 \%$, humedad 6\%, Lisina 1,4 g/100 g, zinc 22,7 mg/100 g, calcio 29013,7 mg/100 g, hierro 277,5 mg/100g, magnesio 1947,8 mg/100 g, Tiamina 0,10 mg/100 g, Riboflavina $0,24 \mathrm{mg} / 100 \mathrm{~g}$, Niacina $0,50 \mathrm{mg} / 100 \mathrm{~g}$, ácido Fólico 0,14 mg/100 g. Se concluyó que el tallo del Silbadero es una fuente significativa de nutrientes, que puede contribuir a suplir necesidades alimentarias.
\end{abstract}

Palabras clave: Geoffroea spinosa Jacq, tallo, Lisina, vitaminas, minerales, vegetales.

\begin{abstract}
The Silbadero (Geoffroea spinosa Jacq) is a legume that is found throughout South American countries such as Chile, Argentina, Bolivia, Uruguay and Colombia. Regarding this species, only taxonomic and phytochemical studies have been reported. The purpose of this study was to determine the nutrient content of the stem of the Silbadero plant. Samples of the species were taken at random through a field trip to the Tres estate, located in Tubara, Colombia. There were determined parameters of proteins, fats, carbohydrates, crude fiber, ash and moisture; also, amino acid lysine; minerals such as zinc, calcium, iron and magnesium and B-complex vitamins (thiamine, riboflavin, niacin, folic acid). Proteins were determined by the Kjeldahl method, fats by the Soxlet method, carbohydrates by difference, crude fiber by acid-basic, ashes by incineration, gravimetric humidity; Lysine by reverse phase chromatography ultrarapid; Minerals by atomic absorption spectrophotometry and vitamins by high performance liquid chromatography. Protein content was $4.56 \%$, fats $0.38 \%$, carbohydrates $37.2 \%$, crude fiber $43.5 \%$, ash $7.6 \%$, moisture $6 \%$, lysine $1.4 \mathrm{~g} / 100 \mathrm{~g}$, zinc $22.7 \mathrm{mg} / 100 \mathrm{~g}$, calcium $29013.7 \mathrm{mg} / 100 \mathrm{~g}$, iron 277.5 $\mathrm{mg} / 100 \mathrm{~g}$, magnesium $1947.8 \mathrm{mg} / \mathrm{100} \mathrm{g}$, Thiamine 0.10 $\mathrm{mg} / 100 \mathrm{~g}$, Riboflavin $0.24 \mathrm{mg} / 100 \mathrm{G}$, Niacin 0.500 .10 $\mathrm{mg} / 100 \mathrm{~g}$, Folic Acid $0.14 \mathrm{mg} / 100 \mathrm{~g}$. It was concluded that Silbadero stem is a significant source of nutrients which can contribute to supplying food needs.
\end{abstract}

Key words: Geoffroea spinosa Jacq, stem, lysine, vitamins, minerals, vegetables.

\footnotetext{
${ }^{*}$ M.Sc. Docente de Tiempo Completo de La Universidad del Atlántico. Grupo de investigación GRIINSAN. Colombia

2 Especialista en Química de Alimentos, Docente de Tiempo Completo de La Universidad del Atlántico. Grupo de investigación GRIINSAN. Colombia

3 Estudiantes de Química y Farmacia Universidad del Atlántico Barranquilla, Colombia. *km 7 vía a Puerto Colombia, celular: 3156877546,amparopua@mail.uniatlantico.edu.co Colombia
} 


\section{INTRODUCCIÓN}

En la especie Geoffroea spinosa Jacq., se han encontrado polisacáridos no fraccionados que contienen bajos niveles de proteínas y altos niveles de carbohidratos, los cuales están compuestos por arabinosa, ramnosa, ácido hexurónico, pequeñas cantidades de galactosa; los carbohidratos tienen una estructura altamente compleja, cada fracción presenta efectos antitrombóticos potentes, lo cual indica que los polisacáridos de estas plantas pueden ser una fuente alternativa de nuevos compuestos anticoagulantes y antitrombóticos (Souza et al. 2015).

Esta especie fue una de las 39 estudiadas en Chaco y Formosa en el norte de Argentina, a las que se les analizaron las propiedades antimicrobianas a partir de los extractos, contra microorganismos tales como Escherichia coli, Salmonella typhimurium, Pseudomonas aeruginosa, Klebsiella peumoniae, Staphylococcus aureus y Enterococcus faecium, se comprobó el poder de inhibición de las especies estudiadas, sobre el crecimiento de estas bacterias (Salvat et al. 2004).

En Colombia, no se han encontrado reportes previos en la literatura científica de la composición de los tallos de Geoffroea spinosa Jacq.

La alimentación es un factor importante para el mantenimiento de la salud, existen estadísticas que citan problemas de salud relacionados con la alimentación: En Colombia la desnutrición global se encuentra en un 3,4\%, con retraso en el crecimiento un 13,2\% (desnutrición crónica), uno de cada seis niños de uno a cuatro años de edad y una de cada seis mujeres embarazadas presentan anemia; con relación a las prácticas de alimentación uno de cada siete colombianos entre 5 y 64 años de edad no consumen carne y huevos diariamente (Profamilia, ICBF, Ministerio de Protección Social 2010). Esta situación indica un preocupante problema de déficit nutricional, lo cual puede deberse al consumo insuficiente de nutrientes (Frank et al. 2008). Por otro lado también se ha encontrado en Colombia, un incremento de enfermedades crónicas no transmisibles, en los últimos años, lo que origina un importante problema de salud pública, lo que se relaciona con inadecuados hábitos alimentarios, elevado consumo de comidas rápidas y bajo consumo de frutas y verduras (Profamilia, ICBF, Ministerio de Protección Social 2010); por estas razones es necesario buscar estrategia para prevenir la carencia de nutrientes, aumentar la disponibilidad y el consumo de los mismos (Cardero et al. 2009), teniendo en cuenta que dentro de los alimentos vegetales, los tallos, tales como el del Silbadero, son perennes todo el año (Sánchez y Barrantes 2008).

Este estudio tiene por objetivo realizar un perfil en el tallo de la especie Geoffroea spinosa Jacq que permita conocer los componentes y posibles aportes nutricionales de esta planta.

\section{MATERIALES Y MÉTODOS}

Se seleccionaron muestras del tallo de Silbadero libres de daños producido por plagas, golpes o puntos negros, en estado de madurez comercial, provenientes de la finca Los Tres ubicada en el Municipio de Tubara, al norte del departamento del Atlántico (Colombia), longitud 7458'43 $\mathrm{O}$, altitud $252 \mathrm{msnm}$, latitud $10^{\circ} 52^{\prime} 32^{\prime \prime} \mathrm{N}$; el material vegetal fue almacenado en bolsas plásticas hasta el laboratorio de Bromatología de La Universidad del Atlántico para realizar parte de las determinaciones analíticas, en donde se sometió a un proceso de limpieza para retirar suciedad, polvo o arena, luego se secó a través de exposición directa al sol por espacio de 10 minutos, se fragmento en porciones pequeñas y se sometió a una molienda y pulverizado, bajo el empleo de un molino eléctrico marca Universal, con el fin de disminuir el tamaño de la partícula del tallo. 
Se llevó a cabo una limpieza previa del material volumétrico para lo cual se empleó una solución de $36 \mathrm{~g}$ de peroxidisulfato amónico en $2,2 \mathrm{~L}$ de ácido sulfúrico al $98 \%$ en un frasco herméticamente cerrado, luego se procedió a enjuagar con abundante agua de grifo $y$ detergente, finalmente se enjuago con agua purificada y se secó en una estufa de desecación.

El material volumétrico (matraces aforados, pipetas volumétricas y buretas) se calibró obteniendo la incertidumbre para cada uno de ellos. Para las determinaciones analíticas se estudiaron dos muestras por triplicado, los análisis proximales se realizaron en el laboratorio de alimentos de la Universidad del Atlántico, los parámetros de vitaminas y minerales se analizaron en el Laboratorio Ortiz Martínez y el aminoácido Lisina en Tecnimicro.

Se empleó el método Kjeldahl, el cual se basa en la destrucción de la materia orgánica con ácido sulfúrico concentrado, formándose sulfato de amonio, que en exceso de hidróxido de sodio libera amoníaco, que se destila en una solución de ácido bórico al 5\% en presencia del indicador de Tashiro (rojo de metilo + azul de metileno) y se valora la solución con ácido sulfúrico 0,1 N (método AOAC 2005).

Luego se calculó el porcentaje de Nitrógeno, para finalmente obtener el porcentaje de proteína con las siguientes ecuaciones:

Porcentaje de Nitrógeno = Volumen de ácido sulfúrico gastado en la titulación * Normalidad del ácido sulfúrico * Meq de Nitrógeno/ Masa de la muestra $* 100 \%$.

Porcentaje de proteína $=$ Porcentaje de Nitrógeno * Factor $(6,25)$.

Se empleó el método Soxhlet, la grasa se extrajo de la muestra utilizando n-hexano como disolvente y fueron determinadas gravimétricamente después de la destilación del n-hexano (método AOAC 2005). El cálculo del porcentaje de grasa se realizó a partir de la siguiente ecuación:
Porcentaje de grasa=Masa final del matraz Masa del matraz vacío/ g muestra * $100 \%$.

Se empleó el método ácido-base, con el uso del equipo Fiwe, el cual se basa en la digestión ácido base utilizando una solución de ácido sulfúrico $1,25 \% \quad \mathrm{p} / \mathrm{v}$ y con una solución de hidróxido de sodio 2,5\% mas alcohol isoamílico como espumante en condiciones específicas. Al residuo se le realizaron unos lavados sucesivos con acetona y agua destilada caliente y fría, se colocaron los crisoles en la estufa de desecación para el secado por una hora a $105{ }^{\circ} \mathrm{C}$ y luego se tara. Finalmente se lleva a la mufla para su incineración durante 4 h, luego se realizaron los cálculos a través de la siguiente ecuación:

Porcentaje de fibra $=$ P1 - P2/P0 * $100 \%$;

Donde P1 es la masa del crisol gouch después de la desecación, P2 es el masa del crisol gouch después de la incineración (método AOAC 2005).

La determinación de cenizas, se llevó a cabo por medio de incineración seca, que consistió en destruir la sustancia orgánica de la muestra problema en la mufla Terrígeno a una temperatura de $550{ }^{\circ} \mathrm{C}$ durante $4 \mathrm{~h}$, hasta obtener una ceniza color gris o gris claro (método AOAC 2005), la ecuación empleada fue la siguiente:

Porcentaje de cenizas $=(\mathrm{P} 2-\mathrm{P} 1) / \mathrm{M} * 100 \%$

Donde P2 es la masa del crisol de porcelana más ceniza, P1 es la masa del crisol de porcelana vacío y $M$ es la masa de la muestra.

Se empleó el método gravimétrico por diferencia de masa del compuesto, que depende de una macromolécula, sometida a un sistema de secado a $105{ }^{\circ} \mathrm{C}$ durante 2 horas, utilizando un equipo analizador de humedad MB35 de halógeno, que registra el porcentaje de humedad. Se empleó el método por diferencia, para determinación de carbohidratos, para esto se sumaron los resultados del contenido de proteínas, grasas, cenizas y humedad, y el 
total se restó de 100, obteniendo el extracto no nitrogenado (método AOAC 2005).

Se utilizó el método de cromatografía liquida de alta resolución (HPLC), las vitaminas Tiamina, Riboflavina, Niacina, ácido Fólico se identificaron por sus tiempos de retención y se cuantificaron comparando las áreas o alturas de los picos problema con los valores obtenidos del análisis patrón. Se emplearon diferentes concentraciones de la muestra para determinar en cuál de las corridas se observaba mejor concentración de las vitaminas (Técnica USP 38 NF 33 2015).

Se empleó el método espectrofotometría de absorción atómica, que transforma la muestra de átomos en estado de vapor y mide la absorción electromagnética emitida o absorbida por dichos átomos. Para esto se realizó una curva de calibración, utilizando concentraciones conocidas de los minerales para el posterior análisis de las muestras, garantizando así la reproducibilidad del método, para esto se empleó un estándar de solución a 1000 ppm de cada uno de los minerales. El equipo utilizado fue Spectraa 240 FS y 240 Z, los resultados fueron expresados en $\mathrm{mg}$ de cada mineral por $\mathrm{g}$ de muestra seca (Técnica USP 38 NF 33 2015).

Se empleó el método cromatografía líquida UFLC (Ultra rápida), la preparación de la muestra se realizó según el método 994,12 de la AOAC, que consiste en hidrolizar las muestras a $110{ }^{\circ} \mathrm{C}$ durante 24 h en ácido clorhídrico 6 N. Se empleó un estándar de Lisina para preparar la solución estándar a un volumen de inyección de 10 $\mu$ l empleando un cromatógrafo marca Shimadzu.

La concentración de Lisina de las muestras se determinó comparando las repuestas obtenidas a partir de la solución muestra con las respuestas obtenidas a partir de la solución estándar.

Para los resultados de los análisis proximales del tallo de Silbadero, se estimaron las medias, con el empleo del programa IBM SPSS Statistics, posteriormente se procedió a realizar comparación de la composición química del tallo de Silbadero con otros tallos comestibles.

\section{RESULTADOS Y DISCUSIÓN}

La composición química de las hortalizas depende del tipo y la procedencia (Astiasarán y Martínez 2002). La tabla 1 muestra los resultados del análisis proximal del tallo de Silbadero comparados con la composición de otros tallos comestibles, en donde se infiere un elevado contenido de proteínas $(4,56 \% \pm 0,06)$, carbohidratos $(37,25 \% \pm 1,19)$ y kilocalorías $(170,14 \pm 0,05)$, con relación a los otros tallos estudiados. Es importante aclarar que los valores de nutrientes referenciados para todos los vegetales de la tabla 1 son vegetales con madurez comercial. Otro aspecto importante a

Tabla 1. Comparación de la composición nutricional del Silbadero con otros tallos comestibles.

\begin{tabular}{lcccccc}
\hline & $\begin{array}{c}\text { Proteína } \\
(\%)\end{array}$ & $\begin{array}{c}\text { Lípidos } \\
(\%)\end{array}$ & $\begin{array}{c}\text { Carbohidratos } \\
(\%)\end{array}$ & $\begin{array}{c}\text { Cenizas } \\
(\%)\end{array}$ & $\begin{array}{c}\text { Humedad } \\
(\%)\end{array}$ & $\begin{array}{c}\text { Energía } \\
\text { Kcal }\end{array}$ \\
\hline Silbadero & 4,56 & $0,38 \pm 0$ & 37,25 & $7,61 \pm 0,18$ & $6,00 \pm 0,36$ & 170,14 \\
Acelga & 0,8 & 0,2 & 4,3 & 2,1 & 94,5 & 13 \\
Apio & 0,7 & 0,1 & 23,5 & 1,2 & 93,7 & 15 \\
Brócoli & 4,0 & 0,3 & 3,7 & 1,2 & 88,9 & 34 \\
Cardo & 1,3 & 0,0 & 2,9 & 0,8 & 94,0 & 17 \\
Cebolla junca & 1,2 & 0,1 & 5,5 & 0,5 & 91,4 & 28 \\
Cebolla puerro & 1,6 & 0,1 & 13,7 & 0,8 & 82,4 & 62 \\
Palmito & 1,6 & 0,2 & 12,5 & 1,5 & 82,8 & 58 \\
Ruibarbo & 0,5 & 0,3 & 3,1 & 0,7 & 94,9 & 15 \\
\hline
\end{tabular}

Fuente: Tabla de Composición Química de Alimentos Colombianos, 2015 
tener en cuenta es que la variación del contenido de nutrientes puede deberse a la genética de las poblaciones de la planta (Atchley 1984).

Con relación al contenido de lípidos, también es ligeramente más elevado que otros tallos

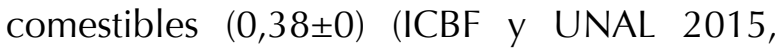
Astiasarán y Martínez 2002), aunque es realmente bajo, aspecto importante para la alimentación, debido a que los alimentos hiperlipídicos presentan una alta densidad calórica (Pinheiro et al. 2008).

El contenido de fibra bruta es elevado en comparación con otros vegetales (Juárez et al. 2012).

Las cenizas expresan el contenido mineral, las cuales se observan por encima de otras especies (Instituto Colombiano de Bienestar Familiar y Universidad Nacional 2015); la humedad es baja, lo que se debe probablemente al lugar donde se cultivó, sin embargo los valores de humedad son similares a otros vegetales (ICBF y UNAL 2015, Centurión et al. 2009).

En la tabla 2 se infiere que dentro de las vitaminas estudiadas en los tallos de Silbadero, el ácido nicotínico o niacina mostró la mayor proporción $(0,50 \mathrm{mg} / 100 \mathrm{~g} \pm 0,01)$, con niveles mínimos de 0,49 mg/100 g hasta 0,51 mg/100 $\mathrm{g}$, no obstante, presenta menor valor que en vegetales como la col y los espárragos

Tabla 2. Comparación del contenido de vitaminas del Silbadero con otros tallos comestibles.

\begin{tabular}{lccc}
\hline & $\begin{array}{c}\text { Tiamina } \\
(\mathrm{mg} / 100 \mathrm{~g})\end{array}$ & $\begin{array}{c}\text { Riboflavina } \\
(\mathrm{mg} / 100 \mathrm{~g})\end{array}$ & $\begin{array}{c}\text { Niacina } \\
(\mathrm{mg} / 100 \mathrm{~g})\end{array}$ \\
\hline Silbadero & 0,10 & 0,24 & 0,50 \\
Acelga & 0,02 & 0,03 & 0,10 \\
Apio & 0,07 & 0,04 & 0,50 \\
Brócoli & 0,07 & 0,14 & 0,90 \\
Cardo & 0,01 & 0,04 & 0,40 \\
Cebolla puerro & 0,07 & 0,05 & 0,50 \\
Palmito & 0,06 & 0,06 & 1,10 \\
Ruibarbo & 0,01 & 0,02 & 0,10 \\
\hline
\end{tabular}

Fuente: Tabla de Composición Química de Alimentos Colombianos, 2015
(Astiasarán y Martínez 2002), acelga, apio, brócoli, cardo, cebolla puerro y ruibarbo (ICBF y UNA 2015), seguidamente se destaca el contenido de Riboflavina $(0,24 \mathrm{mg} / 100 \mathrm{~g}$ $\pm 0,01)$, el ácido Fólico $(0,146 \mathrm{mg} / 100 \pm 0,007)$ y la Tiamina $(0,10 \mathrm{mg} / 100 \mathrm{~g} \pm 0,004)$; el contenido de Riboflavina y de Tiamina es superior a otras especies vegetales comestibles (Meléndez et al. 2013). No se encontraron reportes del contenido de ácido fólico en los otros tallos estudiados, por lo que se recomienda continuar con investigaciones en este campo.

En la tabla 3 se observa el contenido de minerales del tallo de Silbadero comparado con otros tallos, el cual puede estar afectado por la fertilidad del suelo, la genética de la planta y el medio en el que crece (García et al. 2010), se observa que en los tallos del Silbadero prevalece el contenido de calcio (29013,71 mg/100 g士13,9), el cual está muy por encima de otros vegetales, al igual que el contenido de magnesio $(1497,80 \mathrm{mg} / 100$ $\mathrm{g} \pm 74,8)$, el hierro $(277,58 \mathrm{mg} / 100 \mathrm{~g} \pm 15,61)$ y el zinc $(22,7 \mathrm{mg} / 100 \mathrm{~g} \pm 4,39)$ (ICBF y UNAL 2015, Meléndez et al. 2013). No se encontraron reportes del contenido de zinc y magnesio en otros tallos.

Las fuentes más importantes de nutrientes inorgánicos se encuentran en los tejidos vegetales verdes y los vegetales frescos

Tabla 3. Comparación del contenido de hierro y calcio, media (mg/100 g) del Silbaderocon otros tallos comestibles.

\begin{tabular}{lcc}
\hline & Hierro & Calcio \\
\hline Silbadero & $277,58 \pm 15,61$ & 29013,71 \\
Acelga & 0,8 & 31 \\
Apio & 0,4 & 42 \\
Brócoli & 1,1 & 106 \\
Cardo & 0,4 & 39 \\
Cebolla junca & 0,5 & 35 \\
Cebolla puerro & 0,8 & 86 \\
Palmito & 4,4 & 40 \\
Ruibarbo & 1,4 & 344 \\
\hline Fuente: Tabla de & Composición & Química de Alimentos \\
Colombianos, 2015 & &
\end{tabular}


son buena fuente de potasio y magnesio (Casabuena y Bourgues-Rodríguez 2001), por lo que se adjudica el contenido de magnesio a la tonalidad verde de los tallos de Silbadero.

Los alimentos de origen vegetal son las principales fuentes de nutrientes para la población mundial, siendo necesarios para el sustento de la salud y el bienestar (Silva y Ortiz 2011).

La planta de Silbadero, pertenece a la famililia Fabaceae, con más de 18.000 especies en 650 géneros ampliamente distribuidos, y sus frutos se consideran botánicamente una leguminosa, alimento caracterizado por su elevado contenido de carbohidratos complejos (fibra dietética, almidón) y proteínas, en especial el aminoácido esencial Lisina y es el principal aminoácido limitante de los cereales, se ha estudiado en las plantas por años con el fin de caracterizar los principales puntos en la regulación de biosíntesis de otros aminoácidos (Guerra et al. 2001), en los tallos de Silbadero, el análisis de Lisina reportó 1,453 g/100 g, el cual se encuentra por encima de lo reportado en otros estudios (Romao et al. 2003).

\section{CONCLUSIÓN}

Los tallos de Silbadero pueden ser considerados vegetales con elevado contenido de proteínas carbohidratos, fibra y calcio, estas características le dan la posibilidad de convertirse en una alternativa de consumo para regímenes alimentarios, para el fomento de hábitos saludables en la alimentación y para el aprovechamiento por parte de la industria alimentaria.

\section{REFERENCIA}

AOAC Official Methods of Analysis International. 2005. Association of Official Analytica Chemists, Maryland USA, 18 the edition.
Astiasarán, I. y Martínez, A. 2002. Alimentos Composición y Propiedades. Mc Graw Hill Interamericana, Madrid, p173.

Atchley, A. 1984. Nutritive value of palms, Principes, 28(3):138-143.

Casabuena, E. y Bourgues-Rodríguez, H. 2001. Los Nutrimentos. Editorial Panamericana, p 449.

Cardero, Y., Sarmiento, R. y Capdesuñer, A. 2009. Importancia del consumo de hierro y vitamina $C$ para la prevención de anemia ferropènica, MEDISAN, 13 (6).

Centurión, D., Alor, M., Espinosa, J., Gómez, E., Solano, M. y Poot, J. 2009. Contenido nutricional de inflorescencias de palmas en la sierra del estado de Tabasco, Revista Universidad y Ciencia, 25(3): 193-199.

Frank, J., Nieves, M., Torres, M., Bernés, Y., Dallan, G., Rodríguez, D. y Mendoza, N. 2008. Anemia, deficiencias de hierro y de vitamina $A$ y helmintiasis en una población rural del estado de Lara, Anales Venezolanos de Nutrición, 21 (2):70-76.

García, M., Cortés, M. y Rodríguez, E. 2010. Evaluación del secado de perejil aplicando técnicas de deshidratación osmótica como pretratamiento, Revista Facultad Nacional de Agronomía, 63(2): 5693-5705.

Guerra, S., Gaziola, S., Lea, P. y Azevedo, R. 2001. Manipulacao de cereais para acúmulo de Lisina emsementes, Revista Scientia agrícola, 58(1): 205211. https://doi.org/10.1590/S010390162001000100032.

Instituto Colombiano de Bienestar Familiar y Universidad Nacional. 2015. Tabla ce Composición Química de Alimentos Colombianos, Colombia p 158. 
Juárez, A., Muñoz, M., Montes, E., Goytia, M., González, V. y Barcena, R. 2012. Composición química de tallos inmaduros de Acanthecereus, spp. E. Hylocereus Undatus (Haw) Britton\& Rose, Revista Fitotécnica mexicana, 35(2): 171-175.

Meléndez, L., Trabanino, F. y Caballero, A. 2013. Tres perspectivas en torno al uso comestible de las inflorescencias de las palmas de pacay (a), chapay (a) en Chiapas, México: enfoques paleoetnobotánico, nutricional y lingüístico, Revista Estudio de cultura maya, 41:175-199.

Pinheiro, A., Canaan, F. y Goncalves, R. 2008. Insulinemia, ingesta alimentaria y metabolismo energético, Revista chilena de nutrición, 5(1): 18-24.

Profamilia, Instituto Nacional de Salud, Bienestar Familiar, Ministerio de Protección Social. 2010. Encuesta Nacional de la Situación Nutricional en Colombia (ENSIN), [Internet]. Disponible: http://www.icbf.gov.co/portal/page/ portal/Descargas 1/Resumenfi.pdf [Diciembre del 2015].

Romao, L., Lopes, J., Miranda, R., Viana, A., De Olivera, F. y Bunzen, S. 2003. Digestibilidade ileal de aminoácidos de alguns alimentos, determinada pela técnica da cánula T simples com supinos, Revista Brasilera de zootecnia, 32(3): 605-614.
Sánchez, F. y Barrantes, J. 2008. Hojas, flores y tallos comestibles no tradicionales en Costa Rica, Rev. Ciencias Sociales, 1(119):137-152.

Silva, J. y Ortiz, R. 2011. Transgenic vegetable Crops: Progress, Potencials and Prospects, in plantbreedingreviews. Ed. Janick, JhonWiley\&Sons, USA.

Souza, R., Assreuy, A., Madeira, J., Chagas, F., Parreiras, L., Santos, G., Mourao, P. and Pereira, M. 2015. Purifiedpolysaccharides of Geoffroea Spinosa barkshave anticoagulant and antithrombotic activities devoid of hemorrhagic risk, Carbohydrate Polymers, 124: 208-215. https://doi.org/10.1016/j. carbpol.2015.01.069.

Salvat, A., Antonacci, L., Fortunato, R., Suarez, E. and Godoy, H. 2004. Antimicrobial activity in methanolic extracts of several plant species from northern Argentina, Phytomedicine, 11 (2):230-234. https:// doi.org/10.1078/0944-7113-00327.

USP Pharmacopeial Convention. 2005. USP 38 NF 33, PHARMABOOKS EDITOR, 3000p. 